\title{
On a Mixed Nonlinear One Point Boundary Value Problem for an Integrodifferential Equation
}

\author{
Said Mesloub \\ Department of Mathematics, College of Sciences, King Saud University, P.O. Box 2455, \\ Riyadh 11451, Saudi Arabia
}

Correspondence should be addressed to Said Mesloub, mesloub@ksu.edu.sa

Received 31 August 2007; Accepted 5 February 2008

Recommended by Martin Schechter

This paper is devoted to the study of a mixed problem for a nonlinear parabolic integro-differential equation which mainly arise from a one dimensional quasistatic contact problem. We prove the existence and uniqueness of solutions in a weighted Sobolev space. Proofs are based on some a priori estimates and on the Schauder fixed point theorem. we also give a result which helps to establish the regularity of a solution.

Copyright (C) 2008 Said Mesloub. This is an open access article distributed under the Creative Commons Attribution License, which permits unrestricted use, distribution, and reproduction in any medium, provided the original work is properly cited.

\section{Introduction}

In this paper, we are concerned with a one-dimensional nonlinear parabolic integrodifferential equation with Bessel operator, having the form

$$
u_{t}-u_{x x}-\frac{1}{x} u_{x}=\frac{d}{d t} \max \left(\int_{0}^{x} \xi u(\xi, t) d \xi, 0\right)+f,
$$

where $(x, t) \in Q_{T}=(0,1) \times(0, T)$.

Well posedness of the problem is proved in a weighted Sobolev space when the problem data is a related weighted space. In [1], a model of a one-dimensional quasistatic contact problem in thermoelasticity with appropriate boundary conditions is given and this work is motivated by the work of Xie [1], where the author discussed the solvability of a class of nonlinear integrodifferential equations which arise from a one-dimensional quasistatic contact problem in thermoelasticity. The author studied the existence, uniqueness, and regularity of solutions. We refer the reader to [1,2], and references therein for additional information. In the present paper, following the method used in [1], we will prove the existence and uniqueness of $W_{\sigma, 2}^{2,1}\left(Q_{T}\right)$ (see below for definition) solutions of a nonlinear parabolic integrodifferential 
equation with Bessel operator supplemented with a one point boundary condition and an initial condition. The proof is established by exploiting some a priori estimates and using a fixed point argument.

\section{The problem}

We consider the following problem:

$$
\begin{gathered}
u_{t}-u_{x x}-\frac{1}{x} u_{x}=\frac{d}{d t} \max \left(\int_{0}^{x} \xi u(\xi, t) d \xi, 0\right)+f, \quad(x, t) \in Q_{T}=(0,1) \times(0, T), \\
u_{x}(1, t)=0, \quad t \in(0, T), \\
u(x, 0)=g(x), \quad x \in(0,1),
\end{gathered}
$$

where $g(x)$ and $f(x, t)$ are given functions with assumptions that will be given later.

In this paper, $\|\cdot\|_{L_{\mu}^{2}\left(Q_{T}\right)}^{2}$ denotes the usual norm of the weighted space $L_{\mu}^{2}\left(Q_{T}\right)$, where we use the weights $\mu=\sigma, \rho$ and $\sigma=x^{2}$ while $\rho=x$. The respective inner products on $L_{\rho}^{2}\left(Q_{T}\right)$ and $L_{\sigma}^{2}\left(Q_{T}\right)$ are given by

$$
(u, v)_{L_{\rho}^{2}\left(Q_{T}\right)}=\int_{Q_{T}} x u v d x d t, \quad(u, v)_{L_{\sigma}^{2}\left(Q_{T}\right)}=\int_{Q_{T}} x^{2} u v d x d t,
$$

Let $W_{\sigma, 2}^{1,0}\left(Q_{T}\right)$ be the subspace of $L^{2}\left(Q_{T}\right)$ with finite norm

$$
\|u\|_{W_{\sigma, 2}^{1,0}\left(Q_{T}\right)}^{2}=\|u\|_{L_{\sigma}^{2}\left(Q_{T}\right)}^{2}+\left\|u_{x}\right\|_{L_{\sigma}^{2}\left(Q_{T}\right)^{\prime}}^{2}
$$

and $V_{\sigma}=W_{\sigma, 2}^{2,1}\left(Q_{T}\right)$ be the subspace of $W_{\sigma, 2}^{1,0}\left(Q_{T}\right)$ whose elements satisfy $u_{t}, u_{x x} \in L_{\sigma}^{2}\left(Q_{T}\right)$. In general, a function in the space $W_{\sigma, p}^{i, j}\left(Q_{T}\right)$, with $i, j$ nonnegative integers possesses $x$-derivatives up to $i$ th order in the $L_{\sigma}^{p}\left(Q_{T}\right)$, and $t$ th derivatives up to $j$ th order in $L_{\sigma}^{p}\left(Q_{T}\right)$. We also use weighted spaces in the interval $(0,1)$ such as $L_{\sigma}^{2}((0,1))$ and $H_{\sigma}^{1}((0,1))$, whose definitions are analogous to the spaces on $Q_{T}$. We set

$$
W_{\sigma, 2}^{0}((0,1))=L_{\sigma}^{2}((0,1)), \quad W_{\sigma, 2}^{1}((0,1))=H_{\sigma}^{1}((0,1)), \quad W_{\sigma, 2}^{0,0}\left(Q_{T}\right)=L_{\sigma}^{2}\left(Q_{T}\right) .
$$

For general references and proprieties of these spaces, the reader may consult [3].

Throughout this paper, the following tools will be used.

(1) Cauchy inequality with $\varepsilon$ (see, e.g., [4]),

$$
|a b| \leq \frac{\varepsilon}{2}|a|^{2}+\frac{1}{2 \varepsilon}|b|^{2}
$$

which holds for all $\varepsilon>0$ and for arbitrary $a$ and $b$.

(2) An inequality of Poincaré type,

$$
\left\|\Im_{x} u\right\|_{L^{2}\left(Q_{T}\right)}^{2}=\left\|\int_{0}^{x} u(\xi, t) d \xi\right\|_{L^{2}\left(Q_{T}\right)}^{2} \leq \frac{1}{2}\|u\|_{L^{2}\left(Q_{T}\right)}^{2},
$$

where $\mathfrak{I}_{x} u=\int_{0}^{x} u(\xi, t) d \xi$ (see [5, Lemma 1]).

(3) The well-known Gronwall lemma (see, e.g., [6, Lemma 7.1].) 
Remark 2.1. The need of weighted spaces here is because of the singular term appearing in the left-hand side of (2.1) and the annihilation of inconvenient terms during integration by parts.

\section{Existence and uniqueness of the solution}

We are now ready to establish the existence and uniqueness of $V_{\sigma}$ solutions of problem (2.1)(2.3). We first start with a uniqueness result.

Theorem 3.1. Let $f \in L_{\sigma}^{2}\left(Q_{T}\right)$ and $g(x) \in W_{\sigma, 2}^{1}((0,1))$. Then problem (2.1)-(2.3), has at most one solution in $V_{\sigma}$.

Proof. Let $u_{1}$ and $u_{2}$ be two solutions of the problem (2.1)-(2.3) and let $\theta(x, t)=w_{1}(x, t)-$ $w_{2}(x, t)$, where

$$
w_{i}(x, t)=\int_{0}^{t} u_{i}(x, \tau) d \tau, \quad i=1,2
$$

then the function $\theta(x, t)$ satisfies

$$
\begin{aligned}
& \left\llcorner\theta=\theta_{t}-\frac{1}{x}\left(x \theta_{x}\right)_{x}=\max \left(\int_{0}^{x} \xi u_{1}(\xi, t) d \xi, 0\right)-\max \left(\int_{0}^{x} \xi u_{2}(\xi, t) d \xi, 0\right),\right. \\
& \theta_{x}(1, t)=0, \\
& \theta(x, 0)=0 .
\end{aligned}
$$

If we denote by

$$
\beta_{i}(x, t)=\max \left(\int_{0}^{x} \xi u_{i}(\xi, t) d \xi, 0\right), \quad i=1,2,
$$

then calculating the two integrals $\int_{Q_{T}} 2 x^{2} \theta \perp \theta d x d t, \int_{Q_{T}} 2 x^{2} \theta_{t} \perp \theta d x d t$, using conditions (3.3), (3.4), and a combining with $-\int_{Q_{T}} 2 x \theta_{x} \mathcal{L} \theta d x d t$, we obtain

$$
\begin{aligned}
& 2\left\|\theta_{t}\right\|_{L_{\sigma}^{2}\left(Q_{T}\right)}^{2}+2\left\|\theta_{x}\right\|_{L_{\sigma}^{2}\left(Q_{T}\right)}^{2}+\left\|\theta_{x}\right\|_{L^{2}\left(Q_{T}\right)}^{2}+\|\theta(\cdot, T)\|_{L_{\sigma}^{2}((0,1))}^{2}+\left\|\theta_{x}(\cdot, T)\right\|_{L_{\sigma}^{2}((0,1))}^{2} \\
& \quad=-2\left(\theta, \theta_{x}\right)_{L_{\rho}^{2}\left(Q_{T}\right)}+2\left(\theta_{t}, \beta_{1}-\beta_{2}\right)_{L_{\sigma}^{2}\left(Q_{T}\right)} 2\left(\theta, \beta_{1}-\beta_{2}\right)_{L_{\sigma}^{2}\left(Q_{T}\right)}-2\left(\theta_{x}, \beta_{1}-\beta_{2}\right)_{L_{\rho}^{2}\left(Q_{T}\right)} .
\end{aligned}
$$

In light of inequalities (2.7) and (2.8), each term of the right-hand side of (3.6) is estimated as follows:

$$
\begin{aligned}
-2\left(\theta, \theta_{x}\right)_{L_{\rho}^{2}\left(Q_{T}\right)} & \leq\|\theta\|_{L_{\sigma}^{2}\left(Q_{T}\right)}^{2}+\left\|\theta_{x}\right\|_{L^{2}\left(Q_{T}\right)^{\prime}}^{2} \\
2\left(\theta, \beta_{1}-\beta_{2}\right)_{L_{\sigma}^{2}\left(Q_{T}\right)} & \leq 4\|\theta\|_{L_{\sigma}^{2}\left(Q_{T}\right)}^{2}+\frac{1}{8}\left\|\theta_{t}\right\|_{L_{\sigma}^{2}\left(Q_{T}\right)^{\prime}}^{2} \\
2\left(\theta_{t}, \beta_{1}-\beta_{2}\right)_{L_{\sigma}^{2}\left(Q_{T}\right)} & \leq\left\|\theta_{t}\right\|_{L_{\sigma}^{2}\left(Q_{T}\right)}^{2}+\frac{1}{2}\left\|\theta_{t}\right\|_{L_{\sigma}^{2}\left(Q_{T}\right)^{\prime}}^{2} \\
-2\left(\theta_{x}, \beta_{1}-\beta_{2}\right)_{L_{\rho}^{2}\left(Q_{T}\right)} & \leq 4\left\|\theta_{x}\right\|_{L_{\sigma}^{2}\left(Q_{T}\right)}^{2}+\frac{1}{8}\left\|\theta_{t}\right\|_{L_{\sigma}^{2}\left(Q_{T}\right)^{\prime}}^{2}
\end{aligned}
$$


Therefore, using inequalities (3.7), we infer from (3.6)

$$
\left\|\theta_{t}\right\|_{L_{\sigma}^{2}\left(Q_{T}\right)}^{2}+\|\theta(\cdot, T)\|_{L_{\sigma}^{2}((0,1))}^{2}+\left\|\theta_{x}(\cdot, T)\right\|_{L_{\sigma}^{2}((0,1))}^{2} \leq 20\|\theta\|_{L_{\sigma}^{2}\left(Q_{T}\right)}^{2}+20\left\|\theta_{x}\right\|_{L_{\sigma}^{2}\left(Q_{T}\right)}^{2} .
$$

By applying Gronwall's lemma to (3.8), we conclude that

$$
\left\|\theta_{t}\right\|_{L_{\sigma}^{2}\left(Q_{T}\right)}^{2}=0
$$

Hence $u_{1}=u_{2}$.

We now prove the existence theorem.

Theorem 3.2. Let $f \in L_{\sigma}^{2}\left(Q_{T}\right)$ and $g(x) \in W_{\sigma, 2}^{1}((0,1))$ be given and satisfying

$$
\|f\|_{L_{\sigma}^{2}\left(Q_{T}\right)}^{2}+\|g\|_{W_{\sigma, 2}^{1}((0,1))}^{2} \leq c_{2}^{2}
$$

for $c_{2}>0$ small enough and that

$$
g_{x}(1)=0
$$

Then there exists at least one solution $u(x, t) \in W_{\sigma, 2}^{2,1}\left(Q_{T}\right)$ of problem (2.1)-(2.3).

Proof. We define, for positive constants $C$ and $D$ which will be specified later, a class of functions $W=W(C, D)$ which consists of all functions $v \in L_{\sigma}^{2}\left(Q_{T}\right)$ satisfying conditions (2.2), (2.3), and

$$
\|v\|_{V_{\sigma}} \leq C, \quad\left\|v_{t}\right\|_{L_{\sigma}^{2}\left(Q_{T}\right)} \leq D
$$

Given $v \in W(C, D)$, the problem

$$
\begin{gathered}
u_{t}-\frac{1}{x}\left(x u_{x}\right)_{x}=J v+f, \quad(x, t) \in Q_{T}, \\
u_{x}(1, t)=0, \quad t \in(0, T), \\
u(x, 0)=g(x), \quad x \in(0,1),
\end{gathered}
$$

where

$$
J v=\frac{d}{d t} \max \left(\int_{0}^{x} \xi v(\xi, t) d \xi, 0\right),
$$

has a unique solution $u \in V_{\sigma}$. We define a mapping $h$ such that $u=h v$.

Once it is proved that the mapping $h$ has a fixed point $u$ in the closed bounded convex subset $W(C, D)$, then $u$ is the desired solution. 
We, first, show that $h$ maps $W(C, D)$ into itself. For this purpose we write $u$ in the form $u=w+\zeta$, where $w$ is a solution of the problem

$$
\begin{gathered}
w_{t}-w_{x x}-\frac{1}{x} w_{x}=J v, \quad(x, t) \in Q_{T}, \\
w_{x}(1, t)=0, \quad t \in(0, T), \\
w(x, 0)=0, \quad x \in(0,1),
\end{gathered}
$$

and $\zeta$ is a solution of the problem

$$
\begin{gathered}
\zeta_{t}-\zeta_{x x}-\frac{1}{x} \zeta_{x}=f(x, t), \quad(x, t) \in Q_{T}, \\
\zeta_{x}(1, t)=0, \quad t \in(0, T), \\
\zeta(x, 0)=g(x), \quad x \in(0,1) .
\end{gathered}
$$

By multiplying (3.15), (3.18), respectively, by the operators, $O_{1} w=2 x^{2} w+2 x^{2} w_{t}-6 x w_{x}$ and $\mathrm{O}_{2} \zeta=2 x^{2} \zeta+2 x^{2} \zeta_{t}-6 x \zeta_{x}$, then integrating over $Q_{T}$, we obtain

$$
\begin{aligned}
& 2(\mathcal{L} w, w)_{L_{\sigma}^{2}\left(Q_{T}\right)}+2\left(\mathcal{L} w, w_{t}\right)_{L_{\sigma}^{2}\left(Q_{T}\right)}-6\left(\mathcal{L} w, w_{x}\right)_{L_{\rho}^{2}\left(Q_{T}\right)} \\
& =2(J v, w)_{L_{\sigma}^{2}\left(Q_{T}\right)}+2\left(J v, w_{t}\right)_{L_{\sigma}^{2}\left(Q_{T}\right)}-6\left(J v, w_{x}\right)_{L_{\rho}^{2}\left(Q_{T}\right)}
\end{aligned}
$$

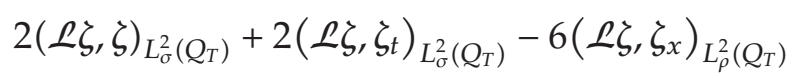

$$
\begin{aligned}
& =2\left(f, \zeta_{t}\right)_{L_{\sigma}^{2}\left(Q_{T}\right)}+2(f, \zeta)_{L_{\sigma}^{2}\left(Q_{T}\right)}-6\left(f, \zeta_{x}\right)_{L_{\rho}^{2}\left(Q_{T}\right)} .
\end{aligned}
$$

By using conditions (3.16), (3.17), (3.19), (3.20), an evaluation of the left-hand side of both equalities (3.21) and (3.22) gives, respectively,

$$
\begin{aligned}
& \|w(x, T)\|_{L_{\sigma}^{2}((0.1))}^{2}+2\left\|w_{x}\right\|_{L_{\sigma}^{2}\left(Q_{T}\right)}^{2}+2\left(w, w_{x}\right)_{L_{\rho}^{2}\left(Q_{T}\right)}+\left\|w_{x}(x, T)\right\|_{L_{\sigma}^{2}((0.1))}^{2} \\
& \quad+2\left\|w_{t}\right\|_{L_{\sigma}^{2}\left(Q_{T}\right)}^{2}+2\left(w_{t}, w_{x}\right)_{L_{\rho}^{2}\left(Q_{T}\right)}+3\left\|w_{x}\right\|_{L^{2}\left(Q_{T}\right)}^{2}-6\left(w_{t}, w_{x}\right)_{L_{\rho}^{2}\left(Q_{T}\right)} \\
& =2(J v, w)_{L_{\sigma}^{2}\left(Q_{T}\right)}+2\left(J v, w_{t}\right)_{L_{\sigma}^{2}\left(Q_{T}\right)}-6\left(J v, w_{x}\right)_{L_{\rho}^{2}\left(Q_{T}\right)^{\prime}}
\end{aligned}
$$

and applying inequalities (2.7), (2.8), and Gronwall's lemma, we obtain the following estimates:

$$
\begin{aligned}
\|\zeta\|_{V_{\sigma}}^{2} & \leq 7 \exp (7 T)\left(\|f\|_{L_{\sigma}^{2}\left(Q_{T}\right)}^{2}+\|g\|_{W_{\sigma, 2}^{1}((0,1))}^{2}\right) \\
& \leq 7 \exp (7 T) c_{2}^{2} \\
\|w\|_{V_{\sigma}}^{2} & \leq 7 \exp (7 T)\|J v\|_{L_{\sigma}^{2}\left(Q_{T}\right)}^{2} .
\end{aligned}
$$


We also multiply by $x$ and square both sides of (3.15), integrate over $Q_{T}$, use the integral $-2 \int_{Q_{T}} x w_{x} \mathcal{L} w d x d t$, then integrate by parts and using inequality (2.7), we obtain

$$
\left\|w_{t}\right\|_{L_{\sigma}^{2}\left(Q_{T}\right)}^{2}+\left\|w_{x x}\right\|_{L_{\sigma}^{2}\left(Q_{T}\right)}^{2}+\left\|w_{x}(\cdot, T)\right\|_{L_{\sigma}^{2}\left(Q_{T}\right)}^{2} \leq 2\|J v\|_{L_{\sigma}^{2}\left(Q_{T}\right)} .
$$

Direct computations yield

$$
\|J v\|_{L_{\sigma}^{2}\left(Q_{T}\right)}^{2} \leq \frac{1}{4}\left(2 c_{1}^{2}+7 \exp (7 T) c_{2}^{2}\right) .
$$

By choosing $c_{1}$ and $c_{2}$ small enough in the previous inequality, we obtain

$$
\|J v\|_{L_{\sigma}^{2}\left(Q_{T}\right)} \leq c_{1}
$$

Inequalities (3.21)-(3.25) then give

$$
\begin{gathered}
\|u\|_{V_{\sigma}}^{2} \leq 2\|w\|_{V_{\sigma}}^{2}+2\|\zeta\|_{V_{\sigma}}^{2} \leq 14 \exp (7 T)\left(c_{2}^{2}+c_{1}^{2}\right) \\
\left\|u_{t}\right\|_{L_{\sigma}^{2}\left(Q_{T}\right)}^{2} \leq 2\left\|w_{t}\right\|_{L_{\sigma}^{2}\left(Q_{T}\right)}^{2}+2\left\|\zeta_{t}\right\|_{L_{\sigma}^{2}\left(Q_{T}\right)}^{2} 4 c_{1}^{2}+14 \exp (7 T) c_{2}^{2}
\end{gathered}
$$

At this point we take $C \geq \sqrt{14} \exp (7 T / 2) \sqrt{\left(c_{1}^{2}+c_{2}^{2}\right)}$ and $D \geq \sqrt{4 c_{1}^{2}+14 \exp (7 T) c_{2}^{2}}$, so that it follows from the last two inequalities that $\|u\|_{V_{\sigma}} \leq C$ and $\left\|u_{t}\right\|_{L_{\sigma}^{2}\left(Q_{T}\right)} \leq D$ from which we deduce that $u \in W=W(C, D)$, hence $h$ maps $W$ into itself. To show that $h$ is a continuous mapping, we consider $v_{1}, v_{2} \in W$ and their corresponding images $u_{1}$ and $u_{2}$. It is straightforward to see that $U=u_{1}-u_{2}$ satisfies

$$
\begin{gathered}
U_{t}-U_{x x}-\frac{1}{x} U_{x}=\frac{d}{d t} \max \left(\int_{0}^{x} \xi v_{1}(\xi, t) d \xi, 0\right)-\frac{d}{d t} \max \left(\int_{0}^{x} \xi v_{2}(\xi, t) d \xi, 0\right), \\
U_{x}(1, t)=0, \quad U(x, 0)=0 .
\end{gathered}
$$

Define the function $p(x, t)$ by the formula

$$
p(x, t)=\int_{0}^{t} U(x, \tau) d \tau,
$$

then it follows from (3.26) and (3.28) that $p(x, t)$ satisfies

$$
\begin{gathered}
p_{t}-p_{x x}-\frac{1}{x} p_{x}=F=\max \left(\int_{0}^{x} \xi v_{1}(\xi, t) d \xi, 0\right)-\max \left(\int_{0}^{x} \xi v_{2}(\xi, t) d \xi, 0\right), \\
p_{x}(1, t)=0, \quad p(x, 0)=0 .
\end{gathered}
$$

Since

$$
\|F\|_{L_{\sigma}^{2}\left(Q_{T}\right)}^{2} \leq\left\|v_{1}-v_{2}\right\|_{L_{\sigma}^{2}\left(Q_{T}\right)^{\prime}}^{2}
$$

then

$$
\|U\|_{L_{\sigma}^{2}\left(Q_{T}\right)}^{2} \leq 6\left\|v_{1}-v_{2}\right\|_{L_{\sigma}^{2}\left(Q_{T}\right)^{\prime}}^{2}
$$


or

$$
\left\|h v_{1}-h v_{2}\right\|_{L_{\sigma}^{2}\left(Q_{T}\right)}^{2} \leq 6\left\|v_{1}-v_{2}\right\|_{L_{\sigma}^{2}\left(Q_{T}\right)^{\prime}}^{2}
$$

hence the continuity of the mapping $h$. The compactness of the set $\overline{W(C, D)}$ is due to the following.

Theorem 3.3. Let $E_{0} \subset E \subset E_{1}$ with compact embedding (reflexive Banach spaces) (see [4, 7]). Suppose that $p, q \in(1, \infty)$ and $T>0$. Then

$$
\Sigma=\left\{\omega: \omega \in L^{p}\left(0, T ; E_{0}\right), \omega_{t} \in L^{q}\left(0, T ; E_{1}\right)\right\}
$$

is compactly embedded in $L^{p}(0, T ; E)$, that is, the bounded sets are relatively compact in $L^{p}(0, T ; E)$.

Note that $L_{\sigma}^{2}\left(0, T ; L_{\sigma}^{2}(0,1)\right)=L_{\sigma}^{2}\left(Q_{T}\right), h(W(C, D)) \subset W(C, D) \subset L_{\sigma}^{2}\left(Q_{T}\right)$. By the Schauder fixed point theorem the mapping $h$ has a fixed point $u$ in $W(C, D)$.

Remark 3.4. For compactness of the set $\overline{W(C, D)}$, see also $[8,9]$.

Remark 3.5. The following theorem gives an a priori estimate which may be used in establishing a regularity result for the solution of (2.1)-(2.3). More precisely, one should expect the solution to be in $W_{\sigma, p}^{2,1}\left(Q_{T}\right)$ with $p \leq \infty$.

Theorem 3.6. Let $u \in V_{\sigma}$ be a solution of problem (2.1)-(2.3), then the following a priori estimate holds:

$$
\begin{aligned}
\sup _{0 \leq t \leq T} & \|u(\cdot, T)\|_{W_{\sigma, 2}^{1}((0,1))}^{2}+\left\|u_{t}\right\|_{L_{\sigma}^{2}\left(Q_{T}\right)}^{2}+\left\|u_{x x}\right\|_{L_{\sigma}^{2}\left(Q_{T}\right)}^{2}+\left\|u_{x}\right\|_{L_{\sigma}^{2}\left(Q_{T}\right)}^{2} \\
& \leq 80 \exp (80 T)\left(\|g\|_{W_{\sigma, 2}^{1}((0,1))}^{2}+\|f\|_{L_{\sigma}^{2}\left(Q_{T}\right)}^{2}\right) .
\end{aligned}
$$

Proof. From (2.1), we have

$$
\begin{aligned}
& \left\|u_{t}\right\|_{L_{\sigma}^{2}\left(Q_{T}\right)}^{2}+\left\|u_{x x}\right\|_{L_{\sigma}^{2}\left(Q_{T}\right)}^{2}+\left\|u_{x}(\cdot, T)\right\|_{L_{\sigma}^{2}((0,1))}^{2}-2\left(u_{t}, u_{x}\right)_{L_{\rho}^{2}\left(Q_{T}\right)} \\
& \quad=\left\|g_{x}\right\|_{L_{\sigma}^{2}((0,1))}^{2}+\int_{Q_{T}} x^{2}\left[\frac{d}{d t} \max \left(\int_{0}^{x} \xi u(\xi, t) d \xi, 0\right)+f\right]^{2} d x d t .
\end{aligned}
$$

Multiplying (2.1) by $2 x^{2} u_{t}$, integrating over $Q_{T}$, carrying out standard integrations by parts, and using conditions (2.2) and (2.3) yields

$$
\begin{aligned}
& 2\left\|u_{t}\right\|_{L_{\sigma}^{2}\left(Q_{T}\right)}^{2}+\left\|u_{x}(\cdot, T)\right\|_{L_{\sigma}^{2}((0,1))}^{2}+2\left(u_{t}, u_{x}\right)_{L_{\rho}^{2}\left(Q_{T}\right)} \\
& \quad=\left\|g_{x}\right\|_{L_{\sigma}^{2}((0,1))}^{2}+2 \int_{Q_{T}} x^{2} u_{t} f d x d t+2 \int_{Q_{T}} x^{2} u_{t} \frac{d}{d t} \max \left(\int_{0}^{x} \xi u(\xi, t) d \xi, 0\right) d x d t .
\end{aligned}
$$


Adding side to side equalities (3.38) and (3.39), then using inequalities (2.7) and (2.8) to estimate the involved integral terms to get

$$
\frac{1}{4}\left\|u_{t}\right\|_{L_{\sigma}^{2}\left(Q_{T}\right)}^{2}+\left\|u_{x x}\right\|_{L_{\sigma}^{2}\left(Q_{T}\right)}^{2}+2\left\|u_{x}(\cdot, T)\right\|_{L_{\sigma}^{2}((0,1))}^{2} \leq 2\left\|g_{x}\right\|_{L_{\sigma}^{2}((0,1))}^{2}+6\|f\|_{L_{\sigma}^{2}\left(Q_{T}\right)}^{2} .
$$

Let be the elementary inequality

$$
\frac{1}{8}\|u(\cdot, T)\|_{L_{\sigma}^{2}((0,1))}^{2} \leq \frac{1}{8}\left\|u_{t}\right\|_{L_{\sigma}^{2}\left(Q_{T}\right)}^{2}+\frac{1}{8}\|u\|_{L_{\sigma}^{2}\left(Q_{T}\right)}^{2}+\frac{1}{8}\|g\|_{L_{\sigma}^{2}((0,1))}^{2} .
$$

Adding the quantity $\left\|u_{x}\right\|_{L_{\sigma}^{2}\left(Q_{T}\right)}^{2}$ to both sides of (3.38), then combining the resulted inequality with (3.39), we obtain

$$
\begin{aligned}
& \|u(\cdot, T)\|_{L_{\sigma}^{2}((0,1))}^{2}+\left\|u_{x}(\cdot, T)\right\|_{L_{\sigma}^{2}((0,1))}^{2}+\left\|u_{t}\right\|_{L_{\sigma}^{2}\left(Q_{T}\right)}^{2}+\left\|u_{x x}\right\|_{L_{\sigma}^{2}\left(Q_{T}\right)}^{2}+\left\|u_{x}\right\|_{L_{\sigma}^{2}\left(Q_{T}\right)}^{2} \\
& \quad \leq 48\left(\|g\|_{W_{\sigma, 2}^{1}((0,1))}^{2}+\|f\|_{L_{\sigma}^{2}\left(Q_{T}\right)}^{2}+\|u\|_{L_{\sigma}^{2}\left(Q_{T}\right)}^{2}+\left\|u_{x}\right\|_{L_{\sigma}^{2}\left(Q_{T}\right)}^{2}\right) .
\end{aligned}
$$

Applying Gronwall's lemma to (3.40) and then taking the supremum with respect to $t$ over the interval $[0, T]$, we obtain the desired a priori bound (3.37).

\section{Acknowledgments}

The author is grateful to the anonymous referees for their helpful suggestions and comments which allowed to correct and improve the paper. This work has been funded and supported by the Research Center Project no. Math/2008/19 at King Saud University.

\section{References}

[1] W. Q. Xie, "A class of nonlinear parabolic integro-differential equations," Differential and Integral Equations, vol. 6, no. 3, pp. 627-642, 1993.

[2] P. Shi and M. Shillor, "A quasistatic contact problem in thermoelasticity with a radiation condition for the temperature," Journal of Mathematical Analysis and Applications, vol. 172, no. 1, pp. 147-165, 1993.

[3] R. A. Adams, Sobolev Spaces, vol. 65 of Pure and Applied Mathematics, Academic Press, New York, NY, USA, 1975.

[4] J.-L. Lions, Équations Différentielles Opérationnelles et Problèmes aux Limites, vol. 111 of Die Grundlehren der mathematischen Wissenschaften, Springer, Berlin, Germany, 1961.

[5] O. A. Ladyzhenskaya, The Boundary Value Problems of Mathematical Physics, vol. 49 of Applied Mathematical Sciences, Springer, New York, NY, USA, 1985.

[6] L. Garding, Cauchy Problem for Hyperbolic Equations, Lecture Notes, University of Chicago, Chicago, Ill, USA, 1957.

[7] J. Simon, "Compact sets in the space $L^{p}(0, T ; B)$, " Annali di Matematica Pura ed Applicata, vol. 146, no. 1, pp. 65-96, 1987.

[8] J.-P. Aubin, “Un théorème de compacité," Comptes Rendus de l'Académie des Sciences, vol. 256, pp. 5042$5044,1963$.

[9] Ju. A. Dubinskiř, "Weak convergence for nonlinear elliptic and parabolic equations," Matematicheskii Sbornik, vol. 67(109), no. 4, pp. 609-642, 1965, (Russian). 